\title{
TOPOLOGICAL PROPERTIES OF GENERALIZED \\ WALLMAN SPACES AND LATTICE RELATIONS
}

\author{
JAMES A. ALLAN \\ Long Island University \\ Department of Mathematics \\ Brooklyn, New York 11201, USA \\ e-mail address: jallan@eagle.liunet edu
}

(Received October 6, 1994 and in revised form March 29, 1995)

\begin{abstract}
Let $X$ be an abstract set and $\mathcal{L}$ be a lattice of subsets of $X$. Associated with the pair $(X, \mathcal{L})$ are a variety of Wallman-type topological spaces. Some of these spaces generalize very important topological spaces such as the Stone-Čech compactification, the real compactification, etc. We consider the general setting and investigate how the properties of $\mathcal{L}$ reflect over to the general Wallman Spaces and conversely. Completeness properties of the lattices in the Wallman Spaces are investigated, as well as the interplay of topological properties of these spaces such as $T_{2}$, regularity and Lindelöf with $\mathcal{L}$.
\end{abstract}

KEY WORDS AND PHRASES. Generalized Wallman Spaces and their topological properties, lattice repleteness and completeness, zero-one valued measures, smoothness properties of measures.

1991 AMS SUBJECT CLASSIFICATION CODES. Primary: 28C15, 28A12; Secondary: 54D60.

\section{INTRODUCTION.}

Let $X$ be an arbitrary non-empty set and $\mathcal{L}$ a lattice of subsets of $X$ such that $\emptyset, X \in \mathcal{L} . \mathcal{A}(\mathcal{L})$ denotes the algebra generated by $\mathcal{L}$, and $I(\mathcal{L})$ denotes the non-trivial zero-one valued finitely additive measures on $\mathcal{A}(\mathcal{L})$. Various specific subsets of $I(\mathcal{L})$ have been considered by researchers along with specific lattices in these subsets together with their topologies of closed sets. They have been referred to as generalized Wallman Spaces (see [3],[4],[5],[6],[8]). Questions concerning their topological properties as well as completeness properties of some of these lattices have also been investigated. The interplay between $\mathcal{L}$ and the topological properties are extremely important. As noted, many specific cases have been considered. We propose here to adopt a very general approach by considering $J(\mathcal{L}) \subset I(\mathcal{L})$ to be any one of the sets usually considered as well as possibly new ones suggested in section 2 , and to consider the lattices of subsets of $J(\mathcal{L})$ as well as the topology of closed sets determined by them. In this general setting, we investigate the interplay between $\mathcal{L}$ and these lattices and topological properties.

In section 2, we review some of the standard notation and terminology and also introduce some new subsets of $I(\mathcal{L})$ for consideration. Section 3 is devoted to an analysis of completeness properties of the lattices of $J(\mathcal{L})$, and also to necessary and sufficient conditions for the topological spaces to be $T_{2}$. Many specific examples are also given.

Section 4 gives a detailed investigation of regularity matters of the lattices and of necessary and sufficient conditions for the spaces to be Lindelöf. Again a large number of examples is presented. 


\section{BACKGROUND AND NOTATION}

In this section, we introduce the notation that will be used throughout the paper, this will be consistent with most of the standard notation used. (See for example [1],[3],[10].) Several items of terminology will also be introduced, all of which are by now quite standard. Finally, we give a brief survey of several of the more important generalized Wallman Spaces (see also [3],[4],[8]), and introduce a few new ones. As indicated in the introduction, it is our aim to systematize the investigation of these spaces by considering a general framework which subsumes all the special cases, and give proofs in this setting which cover the known topological properties of these generalized spaces. Again let $X$ be an arbitrary set and let $\mathcal{L}$ be a lattice of subsets of $X$ such that $\emptyset, X \in \mathcal{L} . \mathcal{L}^{\prime}$ denotes the complementary lattice to $\mathcal{L}$, that is $\mathcal{L}^{\prime}=\left\{L^{\prime} \mid L \in \mathcal{L}\right\}$ where $L^{\prime}=X-L$. Let $I(\mathcal{L})$ be the set of finitely additive nontrivial zero-one valued measures defined on $\mathcal{A}(\mathcal{L})$, the algebra generated by $\mathcal{L}$. We now quickly specify the following subsets of $I(\mathcal{L})$

$I_{R}(\mathcal{L})$ designates the set of $\mu \in I(\mathcal{L})$ which are $\mathcal{L}$-regular, that is $\mu \in I_{R}(\mathcal{L})$ if for all $A \in \mathcal{A}(\mathcal{L})$ we have $\mu(A)=\sup \{\mu(L) \mid L \in \mathcal{L}, L \subset A\} . \quad I_{\sigma}(\mathcal{L})$ denotes the set of $\mu \in I(\mathcal{L})$ which are $\sigma$-smooth on $\mathcal{L}$, that is $\mu \in I_{\sigma}(\mathcal{L})$ if $L_{n} \downarrow \emptyset, L_{n} \in \mathcal{L}$ implies $\mu\left(L_{n}\right) \rightarrow 0 . \quad I^{\sigma}(\mathcal{L})$ are the elements of $I(\mathcal{L})$ that are $\sigma$ smooth on $\mathcal{A}(\mathcal{L})$, that is, the countably additive 0-1 valued measures. $I^{(\sigma)}(\mathcal{L})$ denotes the strongly $\sigma$ smooth measures of $I(\mathcal{L})$; namely $\mu \in I^{(\sigma)}(\mathcal{L})$ if $L_{n} \downarrow L$, with $L_{n}, L \in \mathcal{L}$ implies $\mu\left(L_{n}\right) \rightarrow \mu(L)$. For any $E \in X$, we define:

$$
\mu^{\prime}(E)=\inf \left\{\mu\left(L^{\prime}\right) \mid E \subset L^{\prime}, L \in \mathcal{L}\right\}
$$

It is easy to see that $\mu^{\prime}$ is $0-1$ valued, $\mu^{\prime}(X)=1, \mu^{\prime}(\emptyset)=0, \mu^{\prime}$ is monotone and finitely subadditive; that is $\mu^{\prime}$ is a finitely subadditive outermeasure.

In terms of $\mu^{\prime}$, we can define $I_{\omega}(\mathcal{L})$ where $\mu \in I_{\omega}(\mathcal{L})$ if $\mu \in I(\mathcal{L})$ and if $\mu\left(L^{\prime}\right)=1$ for $L \in \mathcal{L}$ implies the existence of an $\bar{L} \subset L^{\prime}, \bar{L} \in \mathcal{L}$ with $\mu^{\prime}(\bar{L})=1 . I_{\omega}(\mathcal{L})$ is frequently referred to as the set of weakly regular measures.

Various completeness and repleteness notions have been considered (see [3],[4],[7]). We first define for any $\mu \in I(\mathcal{L})$ the support of $\mu$ to be $\mathcal{S}(\mu)=\cap\{L \in \mathcal{L} \mid \mu(L)=1\}$. With this concept, we then have that $\mathcal{L}$ is replete if for any $\mu \in I_{R}^{\sigma}(\mathcal{L}), \mathcal{S}(\mu) \neq \emptyset ; \mathcal{L}$ is prime complete if for any $\mu \in I_{\sigma}(\mathcal{L})$, $\mathcal{S}(\mu) \neq \emptyset ; \mathcal{L}$ is weakly prime complete if for any $\mu \in I^{(\sigma)}, \mathcal{S}(\mu) \neq \emptyset ; \mathcal{L}$ is fully replete if for any $\mu \in I^{\sigma}(\mathcal{L}), \mathcal{S}(\mu) \neq \emptyset$. We also recall that $\pi \in \Pi(\mathcal{L})$ if $\pi: \mathcal{L} \rightarrow\{0,1\}$, and that $\pi(X)=1, \pi(\emptyset)=0, \pi$ is monotone, and $\pi(A \cap B)=\pi(A) \pi(B), A, B \in \mathcal{L}$. $\mathcal{S}(\pi)$, the support of $\pi$, is defined in the obvious way. Also $\pi \in \Pi_{\sigma}(\mathcal{L})$ if $\pi \in \Pi(\mathcal{L})$ and if it is $\sigma$-smooth on $\mathcal{L}$.

There are a number of well-known lattice-topological properties such as disjunctiveness, normality, compactness, etc. (see [5],[7] for further details). We make use of these properties, in particular, their measure theoretic equivalents which will be used throughout sections 3 and 4 . $\delta(\mathcal{L})$ denotes the lattice of countable intersections of sets of $\mathcal{L}$. $\mathcal{L}$ is a delta lattice if and only if $\delta(\mathcal{L})=\mathcal{L}$, that is $\mathcal{L}$ is closed under countable intersections. Finally $\tau(\mathcal{L})$ denotes the lattice of arbitrary intersections of $\mathcal{L}$ sets. Further related matters can be found in ([5],[6],[7],[8],[9]). The various sets of measures that have been introduced can be topologized by taking suitable bases for the closed sets. For example, for the set $I_{R}(\mathcal{L})$, we take $W(\mathcal{L})=\{W(L) \mid L \in \mathcal{L}\}$, where in general

$$
W(A)=\left\{\mu \in I_{R}(\mathcal{L}) \mid \mu(A)=1\right\}
$$

where $A \in \mathcal{A}(\mathcal{L})$, as a base for the closed sets $\tau W(\mathcal{L})$. Special cases of this where $X$ is a topological space and $\mathcal{L}$ a suitable topological lattice yield well-known examples such as the Stone-Čech compactification, the Wallman compactification and the Banachewski compactification. Similarly we can consider $I(\mathcal{L})$ with $V(\mathcal{L})=\{V(L) \mid L \in \mathcal{L}\}$ and where $V(A)=\{\mu \in I(\mathcal{L}) \mid \mu(A)=1\}$, where $A \in \mathcal{A}(\mathcal{L})$, as a base for the closed sets. Frequently, topological properties of these spaces reflect over 
to the original lattice and conversely. for example the space $\langle I(\mathcal{L}), \tau V(\mathcal{L})\rangle$ is always compact and $T_{0}$, it is $T_{1}$ if and only if $I(\mathcal{L})=I_{R}(\mathcal{L})$ which, in turn, is equivalent to $\mathcal{L}=\mathcal{L}^{\prime}$, that is $\mathcal{L}$ is an algebra Also $V(\mathcal{L})$ is regular or disjunctive if and only if $\mathcal{L}=\mathcal{L}^{\prime} \quad$ The Wallman space $I_{R}^{o}(\mathcal{L})$ with $W_{\sigma}(\mathcal{L})$ as base for the closed sets where

$$
W_{o}(\mathcal{L})=\left\{W_{o}(L) \mid L \in \mathcal{L}\right\}
$$

and where $W_{\sigma}(A)=\left\{\mu \in I_{R}^{o}(\mathcal{L}) \mid \mu(A)=1\right\}, A \in \mathcal{A}(\mathcal{L})$, and where $\mathcal{L}$ is disjunctive, is important since it generalizes the real compactification of a topological space Necessary and sufficient conditions for this space to be Lindelof are known in terms of $\mathcal{L}$, and again, it is matters of this type that we wish to generalize

The set $I^{(\sigma)}(\mathcal{L})$ with $V^{(\sigma)}(\mathcal{L})$ as a base where

$$
V^{(\sigma)}(A)=\left\{\mu \in I^{(\sigma)}(\mathcal{L}) \mid \mu(A)=1\right\}
$$

and

$$
V^{(\sigma)}(\mathcal{L})=\left\{V^{(\sigma)}(L) \mid L \in \mathcal{L}\right\}
$$

has not been considered but it can be treated in a way similar to the others It is easy to show that the lattice $V^{(\sigma)}(\mathcal{L})$ is weakly prime complete, and it is prime complete if and only if for any $\mu \in I^{(\sigma)}(\mathcal{L})$ there exists $\gamma \in I^{(\sigma)}(\mathcal{L})$ such that $\mu \leq \gamma(\mathcal{L})$. Also one can give necessary and sufficient conditions for this space to be Lindelöf which are similar to the case of $I_{R}^{\sigma}(\mathcal{L})$ and $W_{\sigma}(\mathcal{L})$ Again it is matters such as these that we want to cover under a general approach which handles all the special cases

As a final particular case, we mention $I_{\omega}(\mathcal{L})$ with $\mathcal{S}(\mathcal{L})$ as a base for the closed sets, where for $A \in \mathcal{A}(\mathcal{L}), \mathcal{S}(A)=\left\{\mu \in I_{\omega}(\mathcal{L}) \mid \mu(A)=1\right\} \quad$ It is not difficult to show that $I_{\omega}(\mathcal{L})$ is then compact, and it is $T_{1}$ if and only if $I_{\omega}(\mathcal{L})=I_{R}(\mathcal{L})$ These types of properties will all be investigated in the next two sections. Further specific generalized Wallman spaces can be found in ([2],[4],[5],[6],[7],[9])

\section{GENERAL STRUCTURE 1}

In this section we begin our unified treatment of generalized Wallman spaces thereby extending the results in ([4],[5],[6],[7],[8])

Again, $X$ is an arbitrary non-empty set, and $\mathcal{L}$ is a lattice of subsets of $X$ We assume for convenience that $\emptyset, X \in \mathcal{L} . \mathcal{A}(\mathcal{L})$ is an algebra generated by $\mathcal{L}$, and $I(\mathcal{L})$ denotes the finitely additive non-trivial, 0-1 valued measures on $\mathcal{A}(\mathcal{L})$. We then designate by $J(\mathcal{L})$ a subset of $I(\mathcal{L})$ In the sequel any $J(\mathcal{L})$ considered will be one of the sets introduced in section 2 , such as $I(\mathcal{L}), I_{R}(\mathcal{L}), I_{\sigma}(\mathcal{L}), I^{\sigma}(\mathcal{L})$, $I_{R}^{\sigma}(\mathcal{L})$, etc We then define $H_{J}(A)=\{\mu \in J(\mathcal{L}) \mid \mu(A)=1\}$ where $A \in \mathcal{A}(\mathcal{L})$. We write $H$ instead of $H_{J}$ when $J$ is fixed. Let $A, B \in \mathcal{A}(\mathcal{L})$, then the following properties are immediate

(1) $H(A \cup B)=H(A) \cup H(B)$.

(2) $H(A \cap B)=H(A) \cap H(B)$.

(3) $H\left(A^{\prime}\right)=H(A)^{\prime}$

(4) $A \subset B \Rightarrow H(A) \subset H(B)$

(5) if $\left\{\mu_{x} \mid x \in X\right\} \subset J(\mathcal{L})$ then $H(A) \subset H(B) \Rightarrow A \subset B$

(6) if $\left\{\mu_{x} \mid x \in X\right\} \subset J(\mathcal{L})$ then $A=B$ if and only if $H(A)=H(B)$

The only one that needs some comment is 5) If $A \not \subset B$, then there exists an $x \in A, x \notin B$ Then $\mu_{x}(A)=1$ and $\mu_{x}(B)=0$ Hence $\mu_{x} \notin H(B)$ but $\mu_{x} \in H(A)$, a contradiction In the case when $J(\mathcal{L})$ is $I_{R}(\mathcal{L})$ or $I_{R}^{\sigma}(\mathcal{L})$ for example, then the condition in 5) and 6) will hold if and only if $\mathcal{L}$ is disjunctive

We note that additional properties hold depending on the nature of $J(\mathcal{L})$ For example if $J(\mathcal{L})=I_{\sigma}(\mathcal{L})$, then $L_{n} \downarrow \emptyset, L_{n} \in \mathcal{L}$, if and only if $H_{J}\left(L_{n}\right) \downarrow \emptyset$; similarly $L_{n} \downarrow L, L_{n}, L \in \mathcal{L}$ if and only if $H_{J}\left(L_{n}\right) \downarrow H_{J}(L)$, if $J(\mathcal{L})=I^{(\sigma)}(\mathcal{L})$, etc 
We assume throughout that $\left\{\mu_{x} \mid x \in X\right\} \subset J(\mathcal{L})$ and consequently the correspondence $\mathcal{L} \rightarrow H(\mathcal{L})$ where $H(\mathcal{L})=\{H(L) \mid L \in \mathcal{L}\}$ is a lattice isomorphism. In the special cases $J(\mathcal{L})=I(\mathcal{L}), I_{R}(\mathcal{L})$, $I_{\sigma}(\mathcal{L}), I^{\sigma}(\mathcal{L}), I^{(\sigma)}(\mathcal{L}), I_{R}^{\sigma}(\mathcal{L})$; and the various $H_{J}(\mathcal{L})$ have been designated in the literature by $V(\mathcal{L})$, $W(\mathcal{L}), V_{\sigma}(\mathcal{L}), V^{\sigma}(\mathcal{L}), V^{(\sigma)}(\mathcal{L}), W_{\sigma}(\mathcal{L})$ respectively (see for example [4]). The above isomorphism enables us to set up a bijection: $J(\mathcal{L}) \rightarrow J(H(\mathcal{L}))$ by mapping $\mu \in J(\mathcal{L})$ onto $\bar{\mu} \in J(H(\mathcal{L})$ ) where $\bar{\mu}(H(A))=\mu(A)$ and where $A \in \mathcal{A}(\mathcal{L})$.

Note clearly that $\mathcal{A}(H(\mathcal{L}))=H(\mathcal{A}(\mathcal{L}))$, and $\bar{\mu}$ is well-defined, since $H(A)=H(B)$ where $B \in \mathcal{A}(\mathcal{L})$ implies $A=B$. The rest of the contention is clear.

Assume now that $\left\{\mu_{x} \mid x \in X\right\} \subset J_{1}(\mathcal{L})$ and $J_{1} \subset J_{2}$. Frequently, in the applications, $J_{2} \subset I_{\sigma}(\mathcal{L})$. Again since $\mathcal{L} \rightarrow H_{J_{1}}(\mathcal{L})$ is a lattice isomorphism, we have a bijection $J_{2}(\mathcal{L}) \leftrightarrow J_{2}\left(H_{J_{1}}(\mathcal{L})\right)$ defined by $\mu \rightarrow \bar{\mu}$, where $\mu \in J_{2}(\mathcal{L})$, and where we define $\bar{\mu}\left(H_{J_{1}}(A)\right)=\mu(A)$ for $A \in \mathcal{A}(\mathcal{L})$. The proof of this is not difficult. We also note that since $J_{1}(\mathcal{L}) \subset J_{2}(\mathcal{L})$, then

$$
J_{1}\left(H_{J_{1}}(\mathcal{L})\right) \subset J_{2}\left(H_{J_{1}}(\mathcal{L})\right) .
$$

We now prove the following:

THEOREM 3.1. With the above notation, we have $\gamma \in \mathcal{S}(\bar{\mu})$ with $\mu \in J_{2}(\mathcal{L})$ and $\gamma \in J_{1}(\mathcal{L})$ if and only if $\mu \leq \gamma(\mathcal{L})$.

PROOF. Suppose $\mu \in J_{2}(\mathcal{L}), \gamma \in J_{1}(\mathcal{L})$ and $\gamma \in \mathcal{S}(\bar{\mu})$. We note that $\mathcal{S}(\bar{\mu})=\cap H_{J_{2}}\left(L_{\alpha}\right)$ where the intersection is taken over those $H_{J_{2}}\left(L_{\alpha}\right), L_{\alpha} \in \mathcal{L}$ such that $\bar{\mu}\left(H_{J_{2}}\left(L_{\alpha}\right)\right)=1$; but this is equivalent to $\mu\left(L_{\alpha}\right)=1$. So $\gamma \in \mathcal{S}(\bar{\mu})$ implies $\gamma\left(L_{\alpha}\right)=1$; hence $\mu \leq \gamma(\mathcal{L})$.

Conversely, suppose for $\mu \in J_{2}(\mathcal{L})$, there exists $\gamma \in J_{1}(\mathcal{L})$ such that $\mu \leq \gamma(\mathcal{L})$. Since $\mathcal{S}(\bar{\mu})=\cap H_{J_{2}}\left(L_{\alpha}\right)$ where $\bar{\mu}\left(H_{J_{2}}\left(L_{\alpha}\right)\right)=1=\mu\left(L_{\alpha}\right) ;$ it follows that $\gamma\left(L_{\alpha}\right)=1$, and clearly $\gamma \in \mathcal{S}(\bar{\mu})$.

We give several specific applications of this theorem, thereby subsuming a large number of known results. See ([4],[5],[6],[8]).

EXAMPLES.

(1) Let $\mathcal{L}$ be disjunctive and consider $J_{1}=I_{R}^{\sigma}, J_{2}=I_{\sigma}, H_{J_{1}}(\mathcal{L})=W_{\sigma}(\mathcal{L})$. Then we have: If $\mu \leq \gamma(\mathcal{L})$ where $\mu \in I_{\sigma}(\mathcal{L}), \gamma \in I_{R}^{\sigma}(\mathcal{L})$, then $\gamma \in \mathcal{S}(\bar{\mu}), \bar{\mu} \in I_{\sigma}\left(W_{\sigma}(\mathcal{L})\right)$. Therefore $W_{\sigma}(\mathcal{L})$ is prime complete (in $I_{R}^{\sigma}$ ) and conversely.

(2) Let $J_{1}=I^{\sigma}, J_{2}=I_{\sigma}$ and $H_{J_{1}}(\mathcal{L})=V^{\sigma}(\mathcal{L})$. Then $\mu \leq \gamma(\mathcal{L}), \mu \in I_{\sigma}(\mathcal{L}), \gamma \in I^{\sigma}(\mathcal{L})$ if and only if $V^{\sigma}(\mathcal{L})$ is prime complete (in $I^{\sigma}(\mathcal{L})$ ).

(3) Let $J_{1}=I^{\sigma}, J_{2}=I^{(\sigma)}$ and $H_{J_{1}}(\mathcal{L})=V^{\sigma}(\mathcal{L})$. Then $\mu \leq \gamma(\mathcal{L}), \mu \in I^{(\sigma)}, \gamma \in I^{\sigma}(\mathcal{L})$ if and only if $V^{\sigma}(\mathcal{L})$ is weakly prime complete (in $I^{\sigma}(\mathcal{L})$ ).

(4) As a special case of Theorem 3.1, we can take $J_{1}=J_{2}=J$, in which case $\mu \in \mathcal{S}(\bar{\mu})$ where $\mu \in J(\mathcal{L})$. This of course implies that $V_{\sigma}(\mathcal{L})$ is prime complete, $V^{(\sigma)}(\mathcal{L})$ is weakly prime complete, $V^{\sigma}(\mathcal{L})$ is fully replete, $V(\mathcal{L})$ is compact, and if $\mathcal{L}$ is disjunctive, $W_{\sigma}(\mathcal{L})$ is replete and $W(\mathcal{L})$ is compact, etc.

We next investigate lattice topological conditions for the lattice $H_{J}(\mathcal{L})$. We continue to assume that $\left\{\mu_{x} \mid x \in X\right\} \subset J(\mathcal{L})$, and we consider the set $J(\mathcal{L})$ and the lattice of subsets of $H_{J}(\mathcal{L})$. The following two theorems show to advantage the general approach and even specific cases do not appear in the references.

THEOREM 3.2. If $I_{R}(\mathcal{L}) \subset J(\mathcal{L})$, then $H_{J}(\mathcal{L})$ is $T_{1}$ if and only if $I_{R}(\mathcal{L})=J(\mathcal{L})$.

PROOF. Suppose $I_{R}(\mathcal{L})=J(\mathcal{L})$, then $W(\mathcal{L})=H_{J}(\mathcal{L})$ and so $H_{J}(\mathcal{L})$ is $T_{1}$ as is well known (see [7]). Conversely suppose $H_{J}(\mathcal{L})$ is $T_{1}$. Let $\mu \in J(\mathcal{L})$; then there exits $\lambda \in I_{R}(\mathcal{L})$ such that $\mu<\lambda(\mathcal{L})$. If $\mu \neq \lambda$, then there exists $L \in \mathcal{L}$ such that $\lambda \in H_{J}(\mathcal{L})^{\prime}, \mu \notin H_{J}(L)^{\prime}$. This implies $\lambda(L)=0, \mu(L)=1$. Therefore $\mu=\lambda \in I_{R}(\mathcal{L})$ and hence $I_{R}(\mathcal{L})=J(\mathcal{L})$. 
By taking $J_{1}(\mathcal{L})=J(\mathcal{L})$ and $J_{2}(\mathcal{L})=I(\mathcal{L})$ in our introductory remarks to this section, we have that $I(\mathcal{L}) \rightarrow I\left(H_{J}(\mathcal{L})\right)$ given $\mu \rightarrow \bar{\mu}$ with $\bar{\mu}\left(H_{J}(A)\right)=\mu(A), A \in \mathcal{A}(\mathcal{L})$ is a bijection $\quad$ Also by Theorem 31 , we have that $\mu \leq \gamma(\mathcal{L})$ where $\mu \in I(\mathcal{L})$ and $\gamma \in J(\mathcal{L})$ is equivalent to $\gamma \in \mathcal{S}(\bar{\mu})$ With these observations, it is now easy to show the following

THEOREM 3.3. Assuming as usual that $\left\{\mu_{x} \mid x \in X\right\} \subset J(\mathcal{L})$ and considering $H_{J}(\mathcal{L})$ in $J(\mathcal{L})$, we have $H_{J}(\mathcal{L})$ is $T_{2}$ if and only if $\mu \in I(\mathcal{L}), \mu \leq \gamma_{1}(\mathcal{L})$ and $\mu \leq \gamma_{2}(\mathcal{L})$ where $\gamma_{1}, \gamma_{2} \in J(\mathcal{L})$ imply $\gamma_{1}=\gamma_{2}$

\section{SPECIAL CASES:}

(1) Let $J=I_{R}$ and let $\mathcal{L}$ be disjunctive, then $H_{J}(\mathcal{L})=W(\mathcal{L})$ Hence $W(\mathcal{L})$ is $T_{2}$ if and only if $\mu \in I(\mathcal{L})$ and $\mu \leq \gamma_{1}(\mathcal{L}), \mu \leq \gamma_{2}(\mathcal{L}), \gamma_{1}, \gamma_{2} \in I_{R}^{\sigma}(\mathcal{L})$ imply $\gamma_{1}=\gamma_{2}$ Thus if $\mathcal{L}$ is disjunctive, then $W(\mathcal{L})$ in $I_{R}(\mathcal{L})$ is $T_{2}$ if and only if $\mathcal{L}$ is normal

(2) Take $J=I^{\sigma}, H_{J}(\mathcal{L})=W(\mathcal{L}) \quad$ Then $W_{\sigma}(\mathcal{L})$ is $T_{2}$ if and only if $\mu \in I(\mathcal{L}), \mu \leq \gamma_{1}(\mathcal{L})$, $\mu \leq \gamma_{2}(\mathcal{L}), \gamma_{1}, \gamma_{2} \in I_{R}^{\sigma}(\mathcal{L})$ implies $\gamma_{1}=\gamma_{2}$

(3) Let $J=I_{\sigma}$ and $H_{J}(\mathcal{L})=V_{\sigma}(\mathcal{L}) \quad$ Then $V_{\sigma}(\mathcal{L})$ is $T_{2}$ if and only if $\mu \in I(\mathcal{L}), \mu \leq \gamma_{1}(\mathcal{L})$, $\mu \leq \gamma_{2}(\mathcal{L}), \gamma_{1}, \gamma_{2} \in I_{\sigma}(\mathcal{L})$ imply $\gamma_{1}=\gamma_{2}$

\section{GENERAL STRUCTURE 2.}

We have considered in section 3 the set $J(\mathcal{L})$ and the lattice of subsets $H_{J}(\mathcal{L})$ Clearly by the basic properties given in section 3 of the mapping $H_{J}$, we can take $H_{J}(\mathcal{L})$ as a base for the closed sets $\tau H_{J}(\mathcal{L})$ of a topology on $J(\mathcal{L})$ Of course $\tau H_{J}(\mathcal{L})$ consists of arbitrary intersections of sets of $H_{J}(\mathcal{L})$ Clearly $H_{J}(\mathcal{L})$ is $T_{2}$ if and only if the topology is a $T_{2}$ topology This is not always the case, lattice topological properties of a lattice do not always extend to the topological lattice $\tau \mathcal{L}$ of closed sets, and conversely. We note, for example, that $\mathcal{L}$ is compact if and only if $\tau \mathcal{L}$ is compact, in which case $\mathcal{L}$ separates $\tau \mathcal{L} . \mathcal{L}$ is countably compact if and only if $\delta(\mathcal{L})$ is countably compact, in which case $\mathcal{L}$ separates $\delta(\mathcal{L})$ However $\mathcal{L}$ being normal need not imply that $\tau \mathcal{L}$ is normal or conversely This will be the case for a pair of lattices $\mathcal{L}_{1} \subset \mathcal{L}_{2}$ if $\mathcal{L}_{1}$ separates $\mathcal{L}_{2}$. If $\mathcal{L}$ is regular then $\tau \mathcal{L}$ is regular, but the converse is not true in general However we do have the following theorem which is not difficult to prove

THEOREM 4.1. If $\tau \mathcal{L}$ is regular and if $\mathcal{L}$ is a delta lattice which is Lindelöf, then $\mathcal{L}$ separates $\tau \mathcal{L}$

We now return to $J(\mathcal{L})$ and the lattice of subsets $H_{J}(\mathcal{L})$. We continue to assume throughout that $\left\{\mu_{x} \mid x \in X\right\} \subset J(\mathcal{L})$. The next theorem again displays the advantage of the general approach. It not only subsumes the special cases in the literature, but gives a systematic and short treatment for all such cases. We show:

THEOREM 4.2. The lattice $H_{J}(\mathcal{L})$ in $J(\mathcal{L})$ is regular if and only if for any $\mu \in I(\mathcal{L}), \mu \leq \rho(\mathcal{L})$ and $\mu \leq \gamma(\mathcal{L})$ where $\mu, \gamma \in I(\mathcal{L})$ and $\rho \in J(\mathcal{L}) \Rightarrow \gamma \leq \rho(\mathcal{L})$.

PROOF. Suppose for any $\mu, \gamma \in I(\mathcal{L})$ and $\rho \in J(\mathcal{L}), \mu \leq \gamma(\mathcal{L})$ and $\mu \leq \rho(\mathcal{L})$ imply $\gamma \leq \rho(\mathcal{L})$ Then for any such $\mu, \gamma$ we have $\bar{\mu}, \bar{\gamma} \in I\left(H_{J}(\mathcal{L})\right)$ and $\bar{\mu} \leq \bar{\gamma}$ on $H_{J}(\mathcal{L})$; this implies that $\mathcal{S}(\bar{\gamma}) \subset \mathcal{S}(\bar{\mu})$ If $\rho \in \mathcal{S}(\bar{\mu})$, then $\rho \in J(\mathcal{L})$ and $\mu \leq \rho(\mathcal{L})$, but $\mu \leq \gamma(\mathcal{L})$; then by the condition of the theorem $\gamma \leq \rho(\mathcal{L})$. Therefore $\rho \in \mathcal{S}(\bar{\gamma})$. Hence $\mathcal{S}(\bar{\gamma})=\mathcal{S}(\bar{\mu})$ and so $H_{J}(\mathcal{L})$ is regular Conversely, suppose $H_{J}(\mathcal{L})$ is regular; let $\mu, \gamma \in I(\mathcal{L})$ and $\rho \in J(\mathcal{L})$ and $\mu \leq \rho(\mathcal{L})$; then $\bar{\mu} \leq \bar{\gamma}$ on $H_{J}(\mathcal{L})$ Therefore $\gamma \leq \rho(\mathcal{L})$.

\section{SPECIAL CASES.}

(1) Let $J(\mathcal{L})=I_{R}^{\sigma}(\mathcal{L})$ where $\mathcal{L}$ is disjunctive Then $H_{J}(\mathcal{L})=W_{\sigma}(\mathcal{L})$ Then $W_{\sigma}(\mathcal{L})$ is regular if and only if for any $\mu \in I(\mathcal{L}), \mu \leq \gamma(\mathcal{L}), \gamma \in I(\mathcal{L})$ and $\mu \leq \rho(\mathcal{L}), \rho \in I_{R}^{\sigma}(\mathcal{L})$ imply $\gamma \leq \rho(\mathcal{L})$.

(2) Let $J(\mathcal{L})=I_{\sigma}(\mathcal{L})$ and $H_{J}(\mathcal{L})=V_{\sigma}(\mathcal{L}) \quad$ Then $V_{\sigma}(\mathcal{L})$ is regular if and only if $\mu, \gamma \in I(\mathcal{L})$, $\rho \in I_{\sigma}(\mathcal{L})$ and $\mu \leq \gamma(\mathcal{L}), \mu \leq \rho(\mathcal{L})$ imply $\gamma \leq \rho(\mathcal{L})$.

(3) Using the theorem we can show that $V_{o}(\mathcal{L})$ is regular if and only if $I_{\sigma}(\mathcal{L})=I_{R}^{\sigma}(\mathcal{L})$ 
Continuing to assume $\left\{\mu_{x} \mid x \in X\right\} \subset J(\mathcal{L})$, we have:

LEMMA 4.3. If $J(\mathcal{L}) \subset I_{\sigma}(\mathcal{L})$ then $L_{n} \downarrow \emptyset, L_{n} \in \mathcal{L}$ is equivalent to $H_{J}\left(L_{n}\right) \downarrow \emptyset$.

PROOF. Suppose $L_{n} \downarrow \emptyset, L_{n} \in \mathcal{L}$; then by the basic properties $H_{J}\left(L_{n}\right)$ is a decreasing sequence of sets and if $\mu \in H_{J}\left(L_{n}\right)$ for all $n$, then $\mu\left(L_{n}\right)=1$ for all $n$. But $\mu \in J(\mathcal{L}) \subset I_{\sigma}(\mathcal{L})$; hence this is a contradiction. Therefore $H_{J}\left(L_{n}\right) \downarrow \emptyset$. Conversely suppose $H_{J}\left(L_{n}\right) \downarrow \emptyset \Rightarrow L_{n}$ is a decreasing sequence of sets; by fundamental properties, if $x \in \cap L_{n}$ for all $n$, then $\mu_{x} \in H_{J}\left(L_{n}\right)$ for all $n$; this is a contradiction. Hence $L_{n} \downarrow \emptyset$.

By the lemma, we get that the correspondence $\pi \rightarrow \bar{\pi}$, where $\pi \in \Pi_{\sigma}(\mathcal{L})$, and where $\bar{\pi}\left(H_{J}(\mathcal{L})\right)=\pi(L)$, for $L \in \mathcal{L}$, is a bijection between $\Pi_{\sigma}(\mathcal{L})$ and $\Pi_{\sigma}\left(H_{J}(\mathcal{L})\right)$. It is now easy to show, abstracting the arguments in ([4],[5]), that the following is true:

THEOREM 4.4. $\mathcal{L}$ satisfies the condition: To each $\pi \in \Pi_{\sigma}(\mathcal{L})$ there exists a $\gamma \in J(\mathcal{L})$ such that $\pi \leq \gamma(\mathcal{L})$ if and only if the topological space $\left\langle J(\mathcal{L}), \tau H_{J}(\mathcal{L})\right\rangle$ is Lindelöf.

\section{SPECIAL CASES.}

(1) Let $\mathcal{L}$ be disjunctive and suppose $J(\mathcal{L})=I_{R}^{\sigma}(\mathcal{L}), \tau H_{J}(\mathcal{L})=\tau W_{\sigma}(\mathcal{L})$. Then $\left\langle I_{R}^{\sigma}(\mathcal{L}), \tau W_{\sigma}(\mathcal{L})\right\rangle$ is Lindelöf if and only if for any $\pi \in \Pi_{\sigma}(\mathcal{L})$ there exists $\gamma \in I_{R}^{\sigma}(\mathcal{L})$ such that $\pi \leq \gamma(\mathcal{L})$.

(2) Suppose $J(\mathcal{L})=I_{\sigma}(\mathcal{L}), \tau H_{J}(\mathcal{L})=\tau V_{\sigma}(\mathcal{L})$. Then $\left\langle I_{\sigma}(\mathcal{L}), \tau V_{\sigma}(\mathcal{L})\right\rangle$ is Lindelöf if and only if for any $\pi \in \Pi_{\sigma}(\mathcal{L})$ there exists $\gamma \in I_{\sigma}(\mathcal{L})$ such that $\pi \leq \gamma(\mathcal{L})$.

ACKNOWLEDGMENT. The author wishes to express his appreciation to the referees for their many helpful comments in the revision of this paper.

\section{REFERENCES}

[1] ALEXANDROFF, A.D., Additive set functions in abstract spaces, Math. Sb. (N.S.), 8, 50 (1940), 307-348.

[2] BACHMAN, G. and STRATIGOS, P.D., Criteria for $\sigma$-smoothness, $\tau$-smoothness and tightness of lattice, regular measures with applications, Canadian Journal of Math., 33, 6 (1981), 1498-1525.

[3] BACHMAN, G. and STRATIGOS, P.D., On general lattice repleteness and completeness, Illinois J. Math., 27, 4 (1983), 535-561.

[4] CONNELL, R., On certain Wallman spaces, International J. Math. Math. Sci., 17, 2 (1994), 273276.

[5] EID, G.M., On Wallman spaces and the Lindelöf property, Annals of NY Acad. of Sci., 659 (1992).

[6] GRASSI, P., Measure characterizations and properties of normal and regular lattices, International J. Math. Math. Sci., 14, 2 (1991), 385-392.

[7] SZETO, M., On separation of lattices, International J. Math. Math. Sci., 14, 2 (1991), 325-338.

[8] VLAD, C., Lattice separation and properties of Wallman-type space, Annali di Mat. Pura ed Appl., 155 (1991), 65-79.

[9] VLAD, C., On normal lattices and semi-separation of lattices, Journal of the Indian Math. Soc., 56 (1991), 259-273.

[10] WALLMAN, H., Lattices and topological spaces, Ann. of Math., 39 (1938), 112-126. 


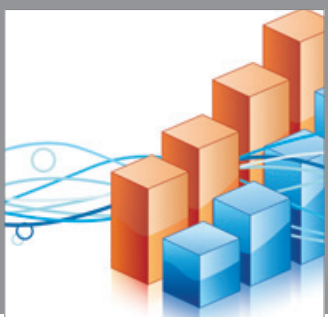

Advances in

Operations Research

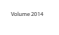

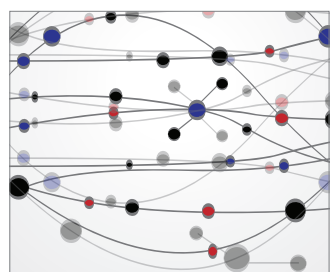

\section{The Scientific} World Journal
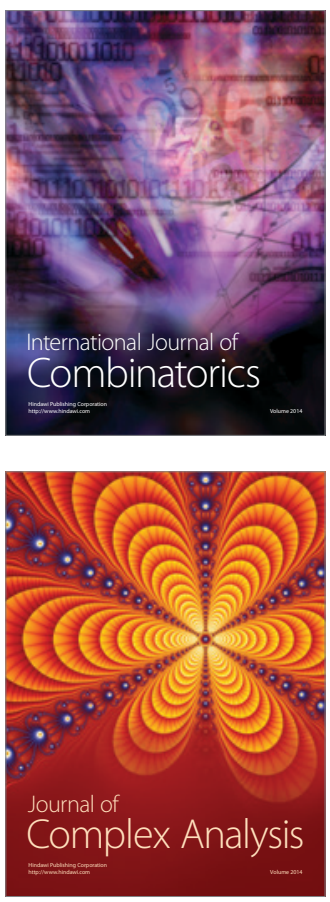

International Journal of

Mathematics and

Mathematical

Sciences
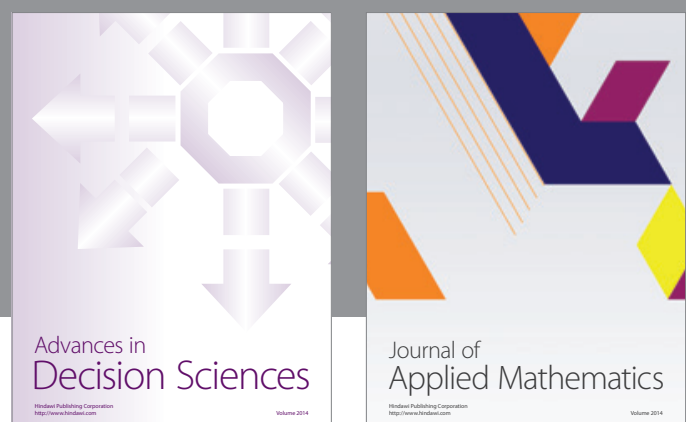

Journal of

Applied Mathematics
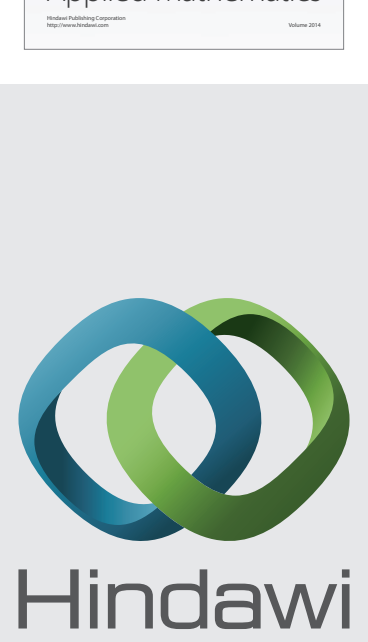

Submit your manuscripts at http://www.hindawi.com
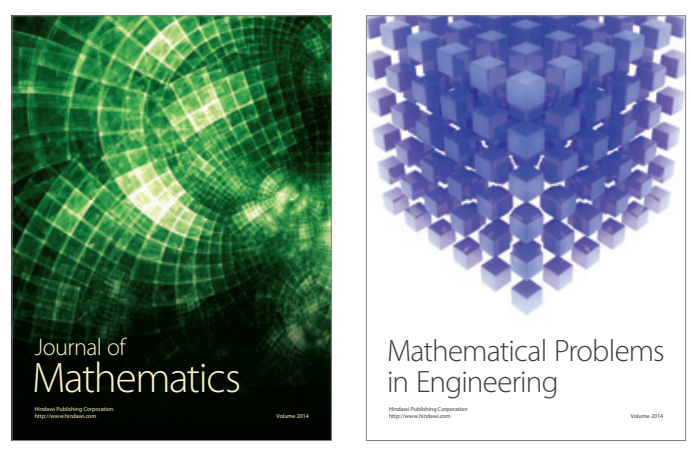

Mathematical Problems in Engineering
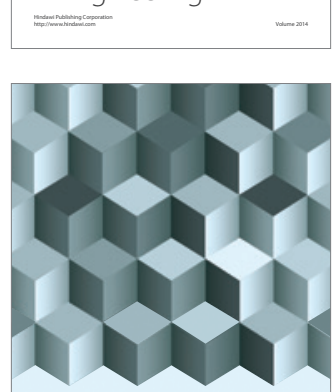

Journal of

Function Spaces
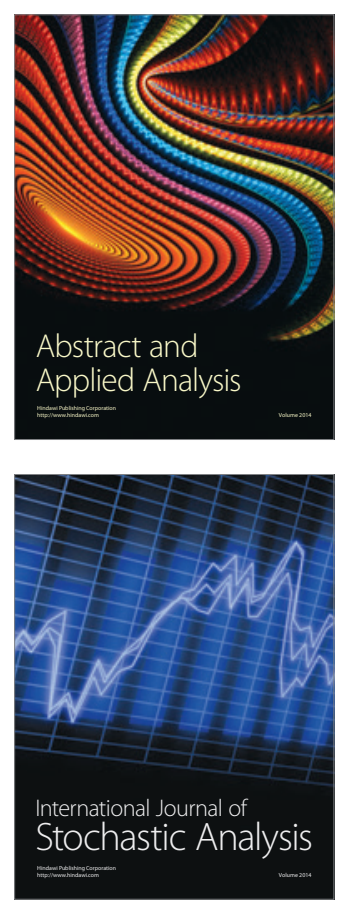

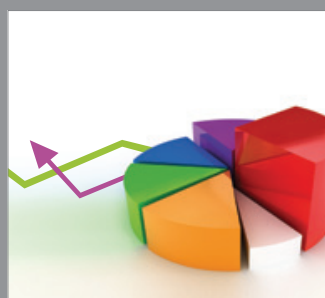

ournal of

Probability and Statistics

Promensencen
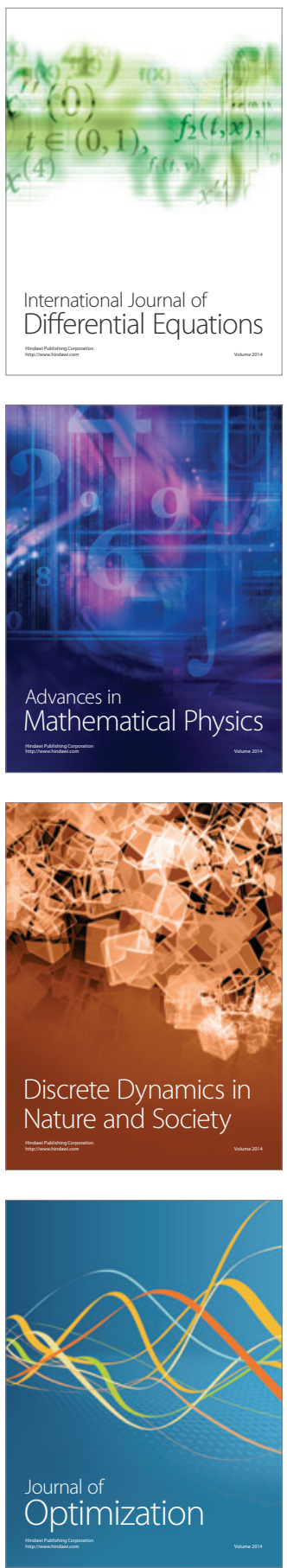